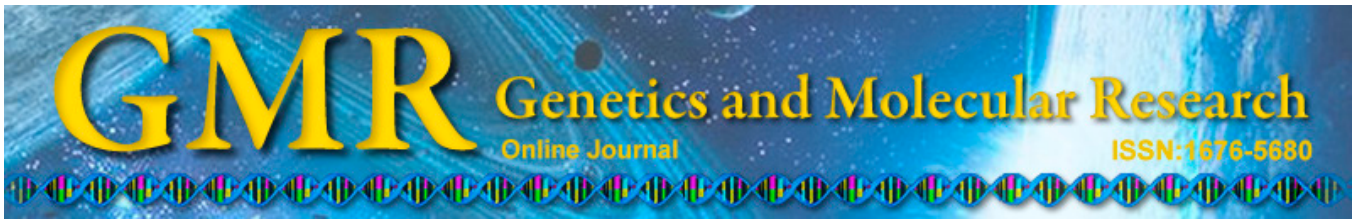

\title{
Clinical image and pathology of hypertrophic cranial pachymeningitis
}

\author{
C.H. Shi ${ }^{1}$, S.T. Niu ${ }^{2}$ and Z.Q. Zhang ${ }^{2}$ \\ ${ }^{1}$ Department of Neurology, Yiwu Central Hospital, Wenzhou Medical College, \\ Yiwu, Zhejiang, China \\ ${ }^{2}$ Department of Neurology, Affiliated Beijing Tiantan Hospital, \\ Capital Medical University, Beijing, China \\ Corresponding author: C.H. Shi \\ E-mail: shichanhong@yeah.net
}

Genet. Mol. Res. 13 (4): 10501-10509 (2014)

Received January 15, 2014

Accepted May 5, 2014

Published December 12, 2014

DOI http://dx.doi.org/10.4238/2014.December.12.11

ABSTRACT. The objective of this study was to examine the clinical findings, magnetic resonance imaging (MRI), pathological features, and treatment experiments of patients with hypertrophic cranial pachymeningitis (HCP). The clinical findings, MRI, and pathological appearances of 9 patients with HCP were analyzed retrospectively. The thickened dura mater was markedly enhanced after contrast media injection. The lesion near the brain hemisphere presented long regions of T1- and T2-weighted abnormal signal intensities. The abnormal signal intensities of the brain tissue were decreased significantly. Pathological examination demonstrated chronic inflammation changes, with cerebral dura mater fibrous tissue showing obvious hyperplasia, and the periphery of the blood vessel showing a great quantity of infiltrating phlegmonosis cells. HCP mainly presents headache and paralysis of multiple cranial nerves. The distinctive signs on brain MRIs involve strengthening the signal in the cerebral dura.

Key words: Hypertrophic cranial pachymeningitis; Clinical image; Magnetic resonance imaging; Pathological features 


\section{INTRODUCTION}

Since 1869 when Charcot first reported hypertrophic pachymeningitis (hypertrophic cranial pachymeningitis, HCP) (Caplan, 1980), the amount of corresponding literature has reached over 200 articles, most of which are from Europe and Japan. HCP is a non-specific chronic inflammatory response in the dura (Martin et al., 1989; Hatano et al., 1999). HCP is a rare disease characterized by diffuse thickening of the dura and the inflammatory process of fibrosis (Sato et al., 1987). The incidence of HCP is low, it has a variety of symptoms, and it is associated with many diseases. It is difficult to get a final diagnosis of HCP in the clinic, and the rate of misdiagnosis of this disease is high.

If proper treatment is not administered, the symptoms of $\mathrm{HCP}$ gradually worsen within a few months, the involvement of the nervous system gradually expands, and the quality of life of patients is seriously affected. If diagnosed early, proper treatment of HCP can improve most clinical symptoms or eliminate them completely; therefore, early diagnosis of $\mathrm{HCP}$ is very important.

We collected 9 cases of patients with HCP, and retrospectively analyzed the associated clinical manifestations, magnetic resonance imaging (MRI) findings, pathological features, and treatment strategies to raise awareness of the disease, improve the diagnosis and proper treatment of the disease, and improve the quality of life of patients with HCP.

\section{MATERIAL AND METHODS}

\section{General information}

Nine patients with HCP were received from Beijing Tiantan Hospital from 2006-2007 to 2009-2010, including 5 males and 4 females, ranging from 25 to 58 years old, with an average age of 42 years. Of these 9 patients, there were 3 cases with acute onset and 6 cases with chronic onset. Symptoms included the following: 9 cases with headache; 9 cases with cranial nerve palsy, including 4 cases with abducens nerve injury; 4 cases with oculomotor impairment; 2, 2, and 2 cases with optic nerve, trigeminal nerve, and auditory nerve injury, respectively; 1 case with trochlear nerve injury; 1 case with facial nerve injury; 2 cases with epilepsy; 1 case with psychopathy; 1 case with fever; 2 cases with a history of otitis media; 1 case with a history of paranasal sinusitis surgery; 1 case with a history of eye tumor surgery; and 1 case with a history of craniocerebral trauma.

In the blood image examination, 3 cases showed a slightly increased number of white blood cells (13.35, 10.37, and 12.02, respectively), 2 cases showed an increased proportion of neutrophils (75 and $82 \%$ ), and 1 case showed a relatively high level of eosinophils (7.30\%). There were no obvious abnormalities in the remaining 5 cases. All 9 patients had normal results for autoantibodies, specific infections (syphilis, etc.), and cancer screening. Three of 9 cases showed a slight increase of erythrocyte sedimentation rate $(26,26$, and 64 , respectively). Seven cases were negative in anti-chain $\mathrm{O}$ and on rheumatoid factor examination. Two of 9 cases showed an increase of lumbar puncture pressure during routine cerebrospinal fluid (CSF) examinations (400 and $270 \mathrm{mmH}_{2} \mathrm{O}$, respectively), and the remaining 7 cases were in the normal range. Three of 9 cases were normal in the CSF routines and biochemical tests, and 6 cases were abnormal to varying degrees. The number of cells was increased in all 6 cases (CSF cell count 16-124/ $\mu \mathrm{L}$ ). Two cases showed an increased protein level (82.90 and $122 \mathrm{mg} /$ 
$\mathrm{dL}$, respectively), and 2 cases showed albuminocytologic dissociation. There were no tumor cells observed in the pathological examination. In the pathogenic examination, we observed 1 case with positive cysticercosis antibody. The remaining 8 cases were negative in CSF smear ink staining, acid-fast stain, Gram stain, and TORCH-IGM, which included toxoplasma gondii, rubella virus, cytomegalovirus, and herpes simplex virus I/II.

\section{Neuroimaging}

Conventional cross-section T1-weighted images (T1WIs) and T2-weighted images (T2WIs), and sagittal and coronal T1WIs were acquired with a 3.0-Tesla superconducting whole-body MRI scanner (SIEMENS, Munich, German). One case with dural lesion was located in the left frontal cortex (Figure 1); 1 dural case involved the bilateral tentorium cerebelli and occipital cerebral falx (Figure 2); 1 case of dural thickening in the tentorium cerebelli and back cranial fossa (Figure 3A); 2 cases of dural in the left temporal and middle cranial fossa (Figure 3B); 2 cases of dural in the tentorium cerebelli and bilateral parasellar cavernous sinus region; and 2 cases of dural in the bilateral tentorium cerebelli, cavernous sinus region, and apical petrous. The T1WIs of the thickened dura showed low or equal signal, while the T2WIs showed significantly low signals with thicknesses of $0.5-2.0 \mathrm{~cm}$, and the dural were mostly striped or patchy. The strength of the dura in the tentorium and falx was special, and showed a central line-like region without enhancement and bilateral orbital-like enhanced regions (Figure 2). It was difficult to distinguish the thickened dura and brain parenchyma in the brain convex on T1WIs, but the difference was easily distinguishable on T2WIs. One case concomitant with the adjacent brain parenchyma lesion manifested flaky hyperintensities on T2WIs and T1WIs. The case also had ipsilateral mastoiditis with irregular enhancement (Figure 3B). Two cases suffered from ipsilateral otitis media and mastoiditis, including 2 cases of prosopantritis that was ipsilateral to the dural lesions in the cavernous sinus, and 1 case with paranasal sinusitis that was ipsilateral to the frontal dural lesions. Significant enhancement of the thickened dura was observed in 8 patients.
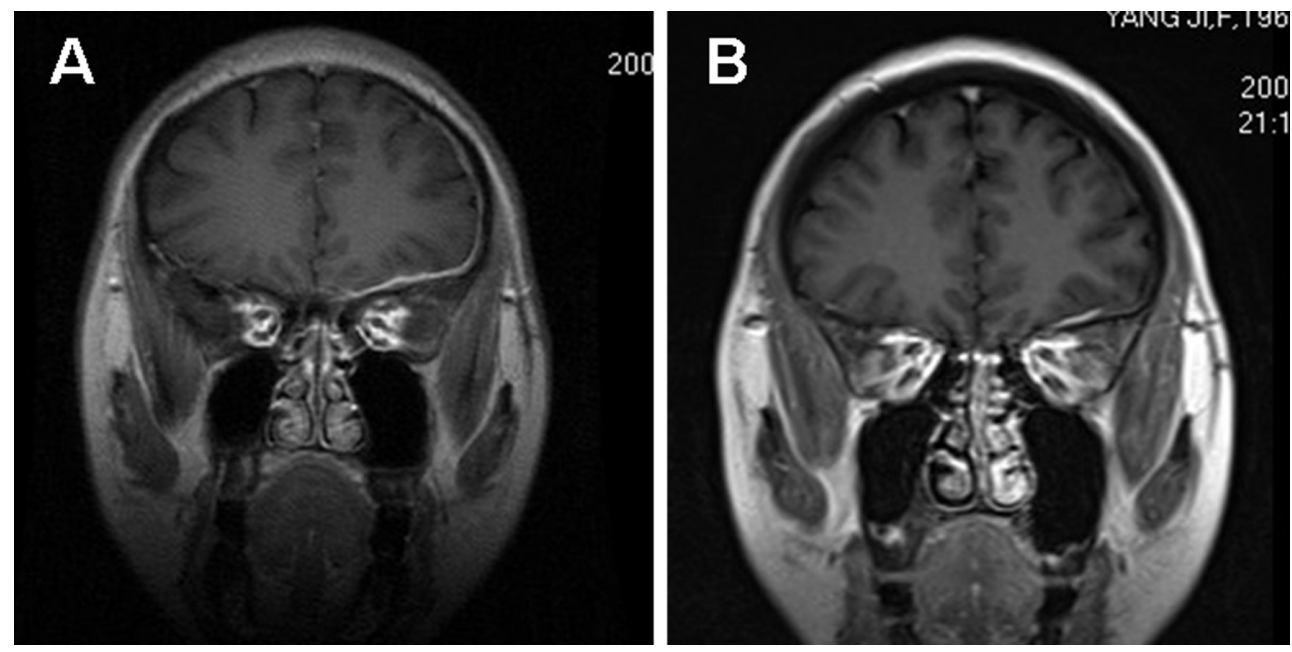

Figure 1. T1WI+C. A. Thickening of left frontal lobe dura. B. No obvious thickening of left frontal lobe dura. 

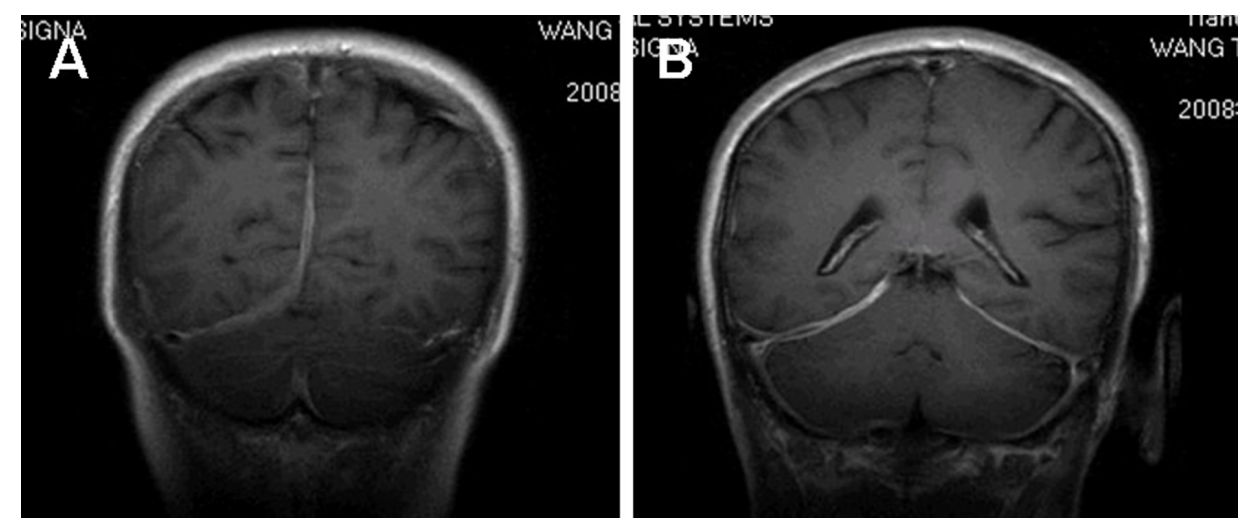

Figure 2. $\mathrm{T} 1 \mathrm{WI}+\mathrm{C}$. A. and B. Bilateral tentorium cerebellum and occipital lobe dura thickening in a strip and plaque form, respectively.
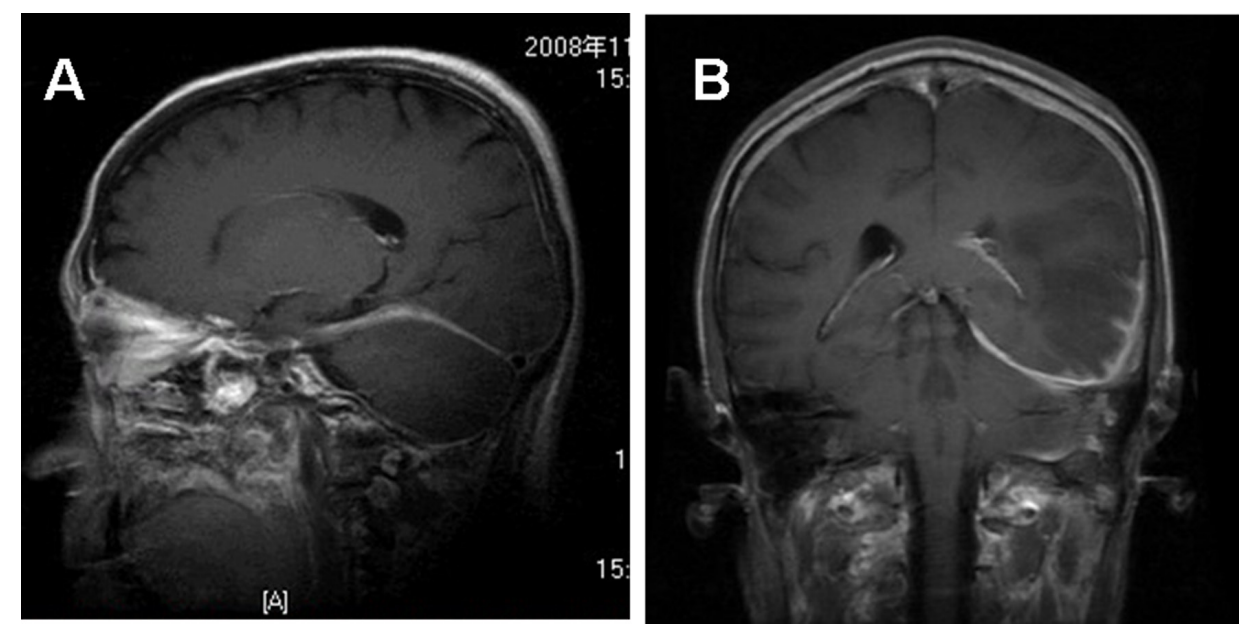

Figure 3. A. $\mathrm{T} 1 \mathrm{WI}+\mathrm{C}$ shows tentorium cerebellum and posterior cranial fossa dura thickening in a strip form. B. T1WI $+\mathrm{C}$ shows thickening of tentorium cerebellum, left temporal lobe, left temporal and parietal lobe dura. Left mastoid process thickening.

\section{Pathological manifestations}

We conducted local dura and multifocal biopsy in 5 patients. In the biopsy, inclusion bodies in amoeba were visible in 1 case and the remaining 4 cases manifested chronic inflammatory changes, plasma cell infiltration, fibrous tissue hyperplasia, hyaline degeneration, or concentric granuloma formation (Figure 4).

\section{Treatment and follow-up}

One patient was treated with oral cyclophosphamide tablets $(150 \mathrm{mg}, 2$ times/week for 40 weeks), and the remaining patients were administered methylprednisolone. After pulse therapy (1000 mg/day) for 3 days, the methylprednisolone was replaced with prednisone (80 
mg every morning). Then, the dose of prednisone was decreased gradually and eventually ceased all together. The treatment lasted for 4-9 months, with an average of 6 months. All patients were treated with a combination of antibiotics ( $1.5 \mathrm{~g}$ cefoperazone sulbactam sodium, 1 time $/ 8 \mathrm{~h} ; 500 \mathrm{mg} /$ day ornidazole) and a neurotrophic agent for 2 weeks to 1 month. The patients were reexamined once a month for 2 years following treatment.

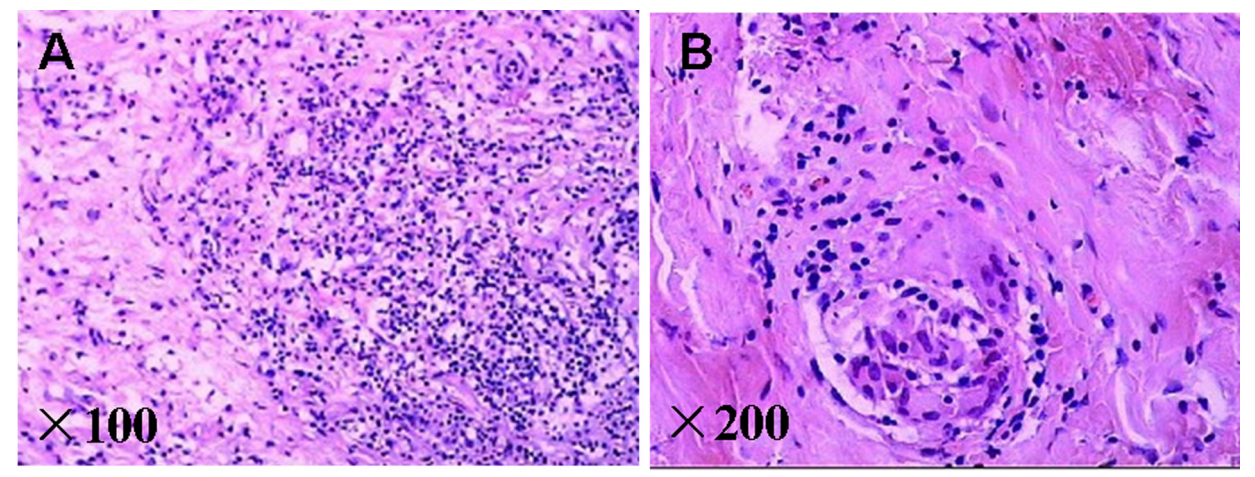

Figure 4. Cerebral dura biopsies. A. Cerebral dura matter fibrous tissue shows obvious hyperplasia. B. Periphery of blood vessel showed a great quantity phlegmonosis cells infiltrating (HE staining). $\mathrm{A}=100 \mathrm{X} ; \mathrm{B}=200 \mathrm{X}$.

\section{RESULTS}

The symptoms of all patients were alleviated after being administered treatment for 2 weeks to 1 month. In the follow-up visits, the symptoms and signs completely disappeared in 5 cases. The headaches and other symptoms disappeared in 4 cases, but some cranial nerve palsy remained. During the treatment process, 4 cases exhibited exacerbations or relapse due to corticosteroid (hereinafter referred to as hormone) reductions, and these symptoms were improved by increasing the hormone dosage. In the MRI reexamination of 7 cases, 6 patients showed some improvement, including thinning of the dura in previously thickened regions, narrowing down and weakening of the enhanced areas (Figure 1), and shrinking or disappearance of the adjacent white matter lesions. The MRIs were reexamined in 2 recurrent cases when their symptoms became exacerbated, with the images showing expansion and thickening of the involved dural areas and enlarging of the white matter edema areas.

\section{DISCUSSION}

\section{The clinical characteristics of HCP}

HCP always occurs in male adults, whose average age is between 40 to 50 years old. Its clinical manifestation is different depending on the occurrence sites. It is generally believed that headaches and cranial nerve damage are the most common clinical manifestations (Hatano et al., 1999). Headaches, often pains in the occipital region of head, can last for years as the only symptom (Wouda and Vanneste, 1998). This may be because of the focal or diffuse dural inflammation. Another reason may be the mild cranial pressure due to dural hypertrophy, or the associated cerebral edema and hydrocephalus. Dural hypertrophy would narrow the gap where there were cranial nerve protrudes. The compression from this 
narrowing can damage the cranial nerve. Damage in cranial nerves I to XII has been reported (Sugiyama et al., 1999; Hamada et al., 2000; Sumida et al., 2000; Yamamoto et al., 2000). The early clinical manifestations include ocular symptoms, such as diplopia, visual acuity loss (Smith and Taxdal, 1966), and even blindness and superior orbital fissure syndrome. Other cranial nerve damage symptoms include: facial pain, tentorium cerebelli, and slope HCP due to trigeminal nerve damage, which can induce damage in cranial nerves V, VII, VIII, IX, and X; Bell's palsy, which is easily misdiagnosed as facial neuritis; and auditory nerve involvement, including dizziness, nausea, vomiting, tinnitus, and even hearing loss. However, the involvement of cranial nerves IX and XII is relatively rare. The cranial nerves that are the most commonly involved are the abducens nerve and oculomotor nerve (4 cases), followed by the optic nerve, trigeminal nerve, auditory nerve ( 2 cases), trochlear nerve, and then the facial nerve (1 case). Brain nerve damage is related to the location of dural involvement. For example, 2 cases with damage to cranial nerves III, IV, and VI were accompanied by visible dural thickening in the ipsilateral cavernous sinus and marked expanded, while 2 otogenic cases were accompanied by visible dural thickening in the ipsilateral petrous bone tip, and these findings are in accordance with previous reports (Swartz et al., 1998). Patients with HCP with partial obvious dural hypertrophy, which can cause compressed brain parenchyma, can present monoplegia, hemiplegia, seizures manifested as generalized tonic-clonic seizures, and partial exercise-induced attacks (Jackson epilepsy) (Bosch et al., 2000; Mizumatsu et al., 2000). Meanwhile, psychiatric symptoms, cerebellar ataxia (Kuhn et al., 2006), intention tremor (Oku et al., 1995), hearing loss, and changes in pituitary function can also occur. Focal cerebral damage is the result of compression from the hypertrophic dura on the adjacent brain tissue and the influence of venous return. In a few cases with venous sinus stenosis caused by dural hypertrophy, some patients may develop dural sinus and venous sinus thrombosis secondarily (Mizumatsu et al., 2000).

\section{Characteristics of imaging and laboratory examinations}

MRI is advantageous because it provides multi-axis imaging, high resolution for soft tissue, and reduces artifacts from the skull base bone. Therefore, it can clearly show the structure of the skull base and has important diagnostic value for HCP (Shindo et al., 1997; Hatano et al., 1999; Sugiyama et al., 1999; Nakazaki et al., 2000). The MRI results were the main basis for diagnosis and follow-up (see the descriptions in the Results). We were able to observe local or diffuse dural hypertrophy by MRI. The lesions showed low or equal signal by T1WIs or T2WIs. However, high signal appeared under contrast-enhanced scan, especially for T1WIs. The increased signal intensity occurs because of the proliferation of fibrous tissue and inflammatory areas. There may be a dural sheath and brain edema around the lesion if the patient has dural hypertrophy. Localized lesions are always located in the tentorium cerebelli, bilateral frontal dura mater, cerebrum falx, as well as a few other regions. Localized dural hypertrophy in the skull base is rare. It may be helpful to discuss the ipsilateral sinusitis or mastoiditis as the pathogenic factors. We can see linear or nodular enhancement in the patients who suffer from diffuse dural hypertrophy. Nodular enhancement represents the unequal dural hypertrophy, which may relate to the degree of repeated dural infection or infection in different parts. This phenomenon suggests poor prognosis. Sometimes the performance of CT and MRI for HCP can be misdiagnosed as subarachnoid hemorrhage. Lumbar puncture can help to diagnose HCP and the results of this treatment appear normal. Some patients may suffer 
from high cerebrospinal fluid pressure, an increase in the number of cells, and a slight increase in protein levels. Additionally, some patients may have erythrocyte sedimentation, slightly elevated C-reactive protein, positive rheumatoid factor-positive, positive core peripheral neutrophils antibody, and so on.

\section{Pathological features of $\mathrm{HCP}$}

HCP was diagnosed according to the pathological examinations. The biopsy of the dura could confirme the diagnosis of HCP. The dural biopsy showed that the fibrous tissue was significantly hyperplastic, manifested a concentric arrangement, and was infiltrated by multiple inflammatory cells (mainly lymphocytes, plasma cells, and fibroblasts). Some of the cases showed chronic non-specific granulomatous hyperplasia, lymphoid follicle formation, hyaline degeneration, caseous necrosis, epithelioid histiocytes, and Langerhans giant cells (Riku and Kato, 2003; Vermeirsch et al., 2006). Granulomatous changes were found in different parts of the dura, suggesting the diffuse infiltration of HCP in the nervous system. Additionally, the dural biopsy could provide a clue for the etiologic diagnosis of HCP, such as whether it is tuberculate, fungal, or amoebic (Takahashi et al., 1998), which could further improve the treatment strategy. A meningeal biopsy is the golden standard of disease confirmation, but there are difficulties that limit the popularity of such a biopsy. Therefore, for most patients, the diagnosis is made based on medical history analysis, clinical features, imaging, and other examinations. It is recommended that a dural biopsy should be considered, particularly when symptoms become aggravated or there are imaging examination changes.

\section{Diagnosis and treatment of HCP}

Clinical findings showed that patients with chronic headache were accompanied by multiple unexplained cranial nerve lesions, in which the diagnosis of HCP should be considered. Idiopathic HCP can be diagnosed using the following steps. Firstly, typical lesions are detected via cranial MRI examination. Secondly, tuberculosis, fungal disease, Lyme disease, and syphilis are excluded using serological tests. Thirdly, lumbar puncture CSF examinations are used to show that infection and tumor-related indices are negative. Idiopathic HCP is relevant to autoimmunity, so it could be treated with corticosteroids and immunosuppressive agents (Shindo et al., 1997; Hatano et al., 1999; Mizumatsu et al., 2000; Nakazaki et al., 2000; Prabhakar et al., 2002). Methylprednisolone, dexamethasone, hydrocortisone, and other corticosteroids are often used in a pulse-tapering way (this therapy could reduce the side effects of drugs). However, premature drug reduction or withdrawal may result in recurrence or aggravation, which occurred in 4 cases in this study. Fortunately, symptoms were alleviated by adjusting hormone dosage, suggesting that the pulse therapy is still effective for the hormone-dependent patients with recurrence. Choi et al. (2000) believe that hormone therapy is effective, but it is easy to relapse and can result in drug dependence. Supplemented with immunosuppressive therapy, high-dose steroid pulse therapy could achieve a satisfactory clinical and imaging result (Rudnik et al., 2007). Cyclophosphamide and methotrexate could be used as immunosuppressive agents, but with significant side effects. These two drugs are ineffective in some patients, which may be caused by a different pathogenesis.

Secondary HCP (Nishioka et al., 1995; Parney et al., 1997) and treatment for the causes should be considered according to both pathological examinations and drug sensitivity 
tests. For example, HCP caused by Aspergillus flavus infection could be treated with itraconazole, amphotericin B, or fluconazole; anti-tuberculosis treatment is required in HCP caused by mycobacterium tuberculosis, and appropriate antibiotics should be used in other bacterial infection-caused HCP. Experimental therapy is feasible if some kind of bacterial infection is suspected without pathological evidence. Generally, medication is effective in secondary HCP, but the prognosis is poor if inflammation still exists. The group of patients in this study was diagnosed with HCP according to their clinical manifestations, MRI, and pathological features and their symptoms were improved via antibiotics and hormone therapy. The treatment outcome was good, even in recurrence cases, according to the follow-up examinations, which were performed once a month for 2 years.

\section{REFERENCES}

Bosch J, Ortega-Aznar A, Tintoré M, Río J, et al. (2000). Hypertrophic pachymeningitis. A review of the histories of two cases and pathological relationship with the Tolosa-Hunt syndrome and the orbital pseudotumor. Rev. Neurol. 31: 946-951.

Caplan LR (1980). "Top of the basilar" syndrome. Neurology 30: 72-79.

Choi IS, Park SC, Jung YK and Lee SS (2000). Combined therapy of corticosteroid and azathioprine in hypertrophic cranial pachymeningitis. Eur. Neurol. 44: 193-198.

Hamada J, Yoshinaga Y, Korogi Y and Ushio Y (2000). Idiopathic hypertrophic cranial pachymeningitis associated with a dural arteriovenous fistula involving the straight sinus: case report. Neurosurgery 47: 1230-1233.

Hatano N, Behari S, Nagatani T, Kimura M, et al. (1999). Idiopathic hypertrophic cranial pachymeningitis: clinicoradiological spectrum and therapeutic options. Neurosurgery 45: 1336-1342; Discussion 1342-1344.

Kuhn J, Harzheim A, Riku S, Müller W, et al. (2006). Hypertrophic cranial pachymeningitis as a rare cause of headache. Nervenarzt 77: 423-429.

Martin N, Masson C, Henin D, Mompoint D, et al. (1989). Hypertrophic cranial pachymeningitis: assessment with CT and MR imaging. AJNR Am. J. Neuroradiol. 10: 477-484.

Mizumatsu S, Michiue H, Suga M, Sunami N, et al. (2000). A case of hypertrophic cranial pachymeningitis developed skull lesion. No To Shinkei 52: 1103-1108.

Nakazaki H, Tanaka T, Isoshima A, Hida T, et al. (2000). Idiopathic hypertrophic cranial pachymeningitis with perifocal brain edema - case report. Neurol. Med. Chir. 40: 239-243.

Nishioka R, Nakajima S, Morimoto Y, Hosoai H, et al.(1995). Hypertrophic cranial pachymeningitis with Propionibacterium acnes detected by dural biopsy. Rinsho Shinkeigaku 35: 526-530.

Oku T, Yamashita M, Inoue T, Sayama T, et al. (1995). A case of posterior fossa hypertrophic pachymeningitis with hydrocephalus. No To Shinkei 47: 569-573.

Parney IF, Johnson ES and Allen PB (1997). "Idiopathic" cranial hypertrophic pachymeningitis responsive to antituberculous therapy: case report. Neurosurgery 41: 965-971.

Prabhakar S, Bhatia R, Lal V and Singh P (2002). Hypertrophic pachymeningitis: varied manifestations of a single disease entity. Neurol. India 50: 45-52.

Riku S and Kato S (2003). Idiopathic hypertrophic pachymeningitis. Neuropathology 23: 335-344.

Rudnik A, Larysz D, Gamrot J, Rudnik A, et al. (2007). Idiopathic hypertrophic pachymeningitis - case report and literature review. Folia Neuropathol. 45: 36-42.

Sato M, Tanaka S and Kohama A (1987). "Top of the basilar" syndrome: clinico-radiological evaluation. Neuroradiology 29: 354-359.

Shindo K, Nitta K, Nagasaka T and Shiozawa Z (1997). Idiopathic hypertrophic cranial pachymeningitis associated with Horner's syndrome. A case report. Rinsho Shinkeigaku 37: 300-303.

Smith JL and Taxdal DS (1966). Painful ophthalmoplegia. The Tolosa-Hunt syndrome. Am. J. Ophthalmol. 61: 14661467.

Sugiyama Y, Shimizu M, Hoshi A, Aoki T, et al. (1999). An old man presenting with fluctuating bilateral multiple cranial nerve palsies and positive test for perinuclear antineutrophil cytoplasmic antibody. No To Shinkei 51: 825-832.

Sumida M, Taguchi H, Eguchi K and Kuroki K (2000). A case of idiopathic cranial hypertrophic pachymeningitis presenting Tolosa-Hunt syndrome. No To Shinkei 52: 523-527.

Swartz JD, Harnsberger HR and Mukherji SK (1998). The temporal bone: contemporary diagnostic dilemmas. Radiol. 
Clin. North Am. 36: 819-853.

Takahashi K, Kobayashi S, Okada K and Yamaguchi S (1998). Pachymeningitis with a perinuclear antineutrophil cytoplasmic antibody: response to pulse steroid. Neurology 50: 1190-1191.

Vermeirsch K, Dubois B, Sciot R and Westhovens R (2006). An external cause for 'idiopathic' pachymeningitis. Acta Clin. Belg. 61: 355-358.

Wouda EJ and Vanneste JA (1998). Aspecific headache during 13 years as the only symptom of idiopathic hypertrophic pachymeningitis. J. Neurol. Neurosurg. Psychiatry 64: 408-409.

Yamamoto T, Goto K, Suzuki A, Nakamura N, et al. (2000). Long-term improvement of idiopathic hypertrophic cranial pachymeningitis by lymphocytapheresis. Ther. Apher. 4: 313-316. 